\title{
X-ray emission from the ultracool dwarf LHS 2065
}

\author{
J. H. M. M. Schmitt and C. Liefke
}

\author{
Hamburger Sternwarte, Universität Hamburg, Gojenbergsweg 112, 21029 Hamburg, Germany \\ e-mail: cliefke@hs.uni-hamburg.de \\ Received 30 July 2001 / Accepted 15 September 2001
}

\begin{abstract}
We report the results of a $68 \mathrm{ks}$ long X-ray observation of the M9V ultracool dwarf star LHS 2065 with the ROSAT high resolution imager (HRI). During the observations a major X-ray flare occurred with a peak X-ray luminosity of $4 \times 10^{27} \mathrm{erg} / \mathrm{s}$ and a total soft energy release of $2 \times 10^{31} \mathrm{erg}$. In addition, another flare with smaller peak X-ray luminosity and energy release occurred. The X-ray observations were carried out half a year apart. In the first half of the observations no significant X-ray emission from LHS 2065 could be detected, while in the second half in addition to flares also quiescent X-ray emission at a level of $2.3 \times 10^{26} \mathrm{erg} / \mathrm{s}$ was seen. LHS 2065 belongs to the coolest hydrogen burning stars known. The ROSAT observations show that coronal emission may be quite common even among such very late-type stars.
\end{abstract}

Key words. stars: activity - stars: coronae - stars: late type - stars: flare - X-rays: stars

\section{Introduction}

During the last few years a great deal of progress has been made in observational studies of very cool stars and brown dwarfs. Increased sensitivity in the infrared, optimal search strategies and improved spectral diagnostics led to the confirmation of the existence of brown dwarfs, i.e., objects below the hydrogen burning mass limit $\left(\approx 0.08 M_{\odot}\right)$, and to the establishment of new spectral classes below M, thus extending the stellar and sub-stellar temperature scale. From the point of view of stellar activity cool $\mathrm{M}$ dwarfs and brown dwarfs are also very intriguing. Upon decreasing the mass of a main sequence star, the star becomes fully convective, yet no obvious sign of any change of activity pattern can be observed. Neither in $\mathrm{H}_{\alpha}$-emission (cf. Giampapa \& Liebert 1986) nor in X-ray emission (cf. Schmitt et al. 1995) does one observe a change in activity related parameters at spectral type $\sim \mathrm{M} 3$, which is thought to indicate fully convective stars. In fact, using $\mathrm{H}_{\alpha}$-emission as an activity indicator, the percentage of active stars increases to essentially one hundred percent at spectral type M7-M9 (Hawley et al. 1996; Gizis et al. 2000). For even cooler stars $\mathrm{H}_{\alpha}$-emission decreases despite evidence for rapid rotation (Basri \& Marcy 1995; Bailer-Jones \& Mundt 1999; Basri 2000) suggesting a break-down of the usual rotationactivity connection. On the other hand, $\mathrm{H}_{\alpha}$-flaring has been reported for quite a number of ultracool stars/brown dwarfs such as BRI0021-0214 (Reid et al. 1999), 2MASS J0149090+295613 (Liebert et al. 1999), and some cool objects such as PC0025+0447 (Schneider et al. 1991) even

Send offprint requests to: J. H. M. M. Schmitt, e-mail: jschmitt@hs.uni-hamburg.de show a persistently high $\mathrm{H}_{\alpha}$-flux. While the connection between time-variable $\mathrm{H}_{\alpha}$-emission and magnetic activity is not compelling for ultracool dwarfs, the data are at least highly suggestive of such a connection.

$\mathrm{H}_{\alpha}$-emission provides evidence for the existence of chromospheres, but not for the existence of coronae. Such evidence is usually derived from X-ray or radio observations. However, X-ray observations often lack sufficient sensitivity. Let us consider a typical ultracool dwarf with a bolometric luminosity of $10^{-3} L_{\odot}$ at a distance of $10 \mathrm{pc}$. Assuming this object to emit X-rays at the saturation level of $L_{\mathrm{X}} / L_{\mathrm{bol}}=10^{-3}$ yields an apparent X-ray flux of $8.4 \times 10^{-14} \mathrm{erg} / \mathrm{cm}^{2} / \mathrm{s}$, which is (usually) not detectable in the ROSAT all-sky survey, but rather easily detectable - for example - in a moderately long ROSAT pointing. However, if the same object were to emit X-rays at solar (maximum) emission levels of $L_{\mathrm{X}} / L_{\mathrm{bol}}=5 \times 10^{-7}$, one computes an apparent X-ray flux of $4.2 \times 10^{-17} \mathrm{erg} / \mathrm{cm}^{2} / \mathrm{s}$, which is detectable (if at all) only in very long pointings with currently available X-ray telescopes. Thus one must keep in mind that even the nearest ultracool dwarfs may exhibit activity levels similar to that observed in the Sun, but still be undetectable with current instrumentation.

Fleming et al. (1993) carried out the first systematic X-ray study of very low mass stars; note that at that time no confirmed brown dwarfs were known. Their "latest" detected star was $\mathrm{vB} 8$, with no detections of $\mathrm{vB} 10$, LHS 2924, and LHS 2065 available. In particular, a long (30 ks) PSPC pointing on LHS 2924 yielded an upper limit of $L_{\mathrm{X}} / L_{\mathrm{bol}}=4.5 \times 10^{-5}$ well below the saturation limit. Since then brown dwarfs in young clusters or star forming regions have been detected as X-ray sources (Neuhäuser \& Comerón 1998; Preibisch \& Zinnecker 2001). As to 
brown dwarfs and ultracool stars in the field, Fleming et al. (2000) report on a long ROSAT HRI observation of vB10, which resulted in a strong flare with a peak X-ray luminosity in excess of $2.7 \times 10^{27} \mathrm{erg} / \mathrm{s}$, but no evidence for any quiescent X-ray emission with an upper limit of $L_{\mathrm{X}} / L_{\mathrm{bol}}<10^{-5}$. Neuhäuser et al. (1999) report an upper limit for the rapidly rotating brown dwarf BRI0021-0214 well below the saturation limit. Rutledge et al. (2000) report an X-ray flare on the brown dwarf LP944-20 with a peak X-ray luminosity of $1.2 \times 10^{26} \mathrm{erg} / \mathrm{s}$ and again no evidence for quiescent $\mathrm{X}$-ray emission with $L_{\mathrm{X}} / L_{\mathrm{bol}}<2 \times 10^{-6}$. Interestingly, Berger et al. (2001) report a radio detection of LP944-20, providing evidence not only for the existence of a corona but also for the existence of relativistic particles in its corona. Thus the available X-ray data also suggest that flaring X-ray emission and hence the existence of - at least transient - coronae is quite common in ultracool low mass stars and brown dwarfs. Quiescent emission is usually not detected (but see Fleming et al. 2001), yet most data are not sensitive enough to exclude solar-like quiescent emission levels.

\section{The ultracool M-dwarf LHS 2065}

Located at a distance of 8.5 pc (Monet et al. 1992), LHS 2065 belongs to the nearest ultracool dwarf stars. Regarding its optical properties (spectral type M9) it is very similar to LHS 2924, which was not detected by Fleming et al. (1993) in a rather deep pointing with the ROSAT PSPC. Martin et al. (1994) failed to detect lithium in the photosphere of LHS 2065, suggesting that the object is old (age $>0.5 \mathrm{Gyr}$ ) and is actually a star rather than a brown dwarf. LHS 2065 does show weak $\mathrm{H}_{\alpha^{-}}$ emission, in addition Martín \& Ardila (2001) report the detection of a strong $\mathrm{H}_{\alpha}$-flare decaying on a time scale of a few hours. Compiling all published literature on LHS 2065 the same authors estimate a flare rate of $<0.03 \mathrm{hr}^{-1}$, while weaker flares maybe quite common.

\subsection{X-ray observations}

Fleming et al. (1993) searched the RASS data for LHS 2065 and obtained an upper limit of $\log L_{\mathrm{X}}<26.57$. Later LHS 2065 was observed with the ROSAT high resolution imager (HRI) for a total of $69.2 \mathrm{ks}$. The observations were carried out in April and May of 1997 (26.3 ks) and at the end of October 1997 (42.9 ks). The data were retrieved from the MPE ROSAT archive (ROR number 202305 ). Before creating images the recorded count events were screened and pulse height channels above 10 were excluded from analysis since they contribute essentially only background. Inspection of the X-ray images showed no clear source in the image constructed from the $26.3 \mathrm{ks}$ exposure taken in April and May 1997, but a clear detection in the October 1997 image.

We then decided to analyze the individual ROSAT observation intervals. ROSAT observations are usually not taken continuously, rather the scientific data stream is interrupted because of earth blocks, passages through the radiation belts and other observations. For each such observation segment we placed a detect cell on the expected source position, determined the number of counts $C_{\mathrm{d}}$ in the detect cell and the background from an adjacent much larger region. The expected background $b_{\mathrm{d}}$ in the detect cell was then extrapolated by scaling the areas of background extraction region and detect cell. Finally we calculated the probability $P$ of obtaining at least the observed detect cell count $C_{\mathrm{d}}$ under the assumption of observing a Poisson process with expectation value $b_{\mathrm{d}}$. The results of this exercise are listed in Table 1 . Note that the calculated probabilities refer to a single observation interval only and do not take into account the fact that we obtained a fair number of such intervals (50). As a control experiment we carried out exactly the same procedure on a random apparently source-free background region. From such control experiments we conclude that probabilities of down to $10^{-3}$ do occur especially in time intervals with measured high background and that therefore probabilities in excess of $10^{-3}$ cannot be considered significant. Inspection of Table 1 shows that there are 9 observation intervals with a single occurrence probability of $<10^{-3}$. None of these occur in data taken in April and May 1997, but note that we chose a larger detect cell because of uncertainties in the true source position. Of these 9 low probability intervals 5 are contiguous between October 30 21:19 and Oct. 31 3:48. The other four instances result from data intervals with 6 counts while expecting 0.76, 9 counts expecting $0.61,5$ counts expecting 0.60 , and 5 counts expecting 0.74 . The five contiguous low probability events have extremely high statistical significance. Inspection of the X-ray image constructed only from photons received during this time interval shows a clear source present at the expected position and we therefore conclude that LHS 2065 was caught in a large X-ray flare. The significance of the other "events" is less clear. We believe that the event on Oct. 28 21:40 with 9 observed counts with 0.61 counts expected is actually another - smaller - X-ray flare. The statistical significance of the other "events" is marginal and we hence refrain from interpreting these any further.

\subsection{X-ray light curve}

The total number of photons attributable to the LHS 2065 flare is $37-1.26=35.74$. With this small number of photons it is obviously difficult to characterize the temporal behavior of the flare emission.g With these caveats in mind we show the X-ray light curve obtained from binning the data in $500 \mathrm{~s}$ bins in Fig. 1. As is clear from Fig. 1, a detection has only been made in two observation intervals, emission in the observing intervals before Oct. 30 22:59 and after Oct. 31 2:01 may be present (cf. Table 1). Inspection of the 25 photons received in the first interval shows that it is likely that the flare actually peaked during the $1433 \mathrm{~s}$ ROSAT observation interval starting on 
Table 1.

\begin{tabular}{|c|c|c|c|c|c|c|}
\hline & Date & $\begin{array}{l}\text { Time } \\
\text { (UT) }\end{array}$ & $\begin{array}{r}\text { Expo. } \\
(\mathrm{s})\end{array}$ & Counts & $\begin{array}{l}\text { Back- } \\
\text { ground }\end{array}$ & $\begin{array}{r}\text { Prob. } \\
\text { log }\end{array}$ \\
\hline 1 & Apr. 21 & $9: 57$ & 2368 & 3 & 4.70 & -0.07 \\
\hline 2 & Apr. 30 & 4:01 & 2310 & 5 & 4.38 & -0.35 \\
\hline 3 & May 1 & $2: 14$ & 2227 & 2 & 3.96 & -0.04 \\
\hline 4 & May 1 & $15: 07$ & 1636 & 9 & 3.78 & -1.81 \\
\hline 5 & May 6 & $8: 20$ & 1411 & 4 & 5.93 & -0.07 \\
\hline 6 & May 6 & $9: 33$ & 1183 & 3 & 2.00 & -0.49 \\
\hline 7 & May 6 & $14: 17$ & 1158 & 1 & 2.15 & -0.05 \\
\hline 8 & May 9 & $17: 08$ & 863 & 6 & 1.88 & -1.90 \\
\hline 9 & May 12 & $15: 10$ & 681 & 1 & 1.18 & -0.16 \\
\hline 10 & May 12 & $16: 47$ & 706 & 1 & 1.44 & -0.12 \\
\hline 11 & May 12 & $18: 21$ & 833 & 1 & 1.71 & -0.09 \\
\hline 12 & May 12 & $19: 55$ & 873 & 2 & 1.50 & -0.35 \\
\hline 13 & May 15 & $5: 38$ & 1665 & 13 & 5.66 & -2.25 \\
\hline 14 & May 15 & $6: 53$ & 870 & 1 & 1.17 & -0.16 \\
\hline 15 & May 15 & $21: 12$ & 1956 & 5 & 3.93 & -0.45 \\
\hline 16 & May 15 & $21: 59$ & 209 & 2 & 0.42 & -1.17 \\
\hline 17 & May 22 & 8:02 & 1815 & 7 & 4.93 & -0.64 \\
\hline 18 & May 23 & $7: 55$ & 1751 & 3 & 4.05 & -0.11 \\
\hline 19 & May 23 & $9: 28$ & 1834 & 10 & 4.95 & -1.52 \\
\hline 20 & Oct. 26 & $6: 01$ & 1262 & 2 & 0.89 & -0.65 \\
\hline 21 & Oct. 26 & $7: 34$ & 1263 & 6 & 0.76 & -3.84 \\
\hline 22 & Oct. 26 & $21: 53$ & 1246 & 2 & 0.69 & -0.82 \\
\hline 23 & Oct. 27 & $2: 42$ & 1206 & 0 & 0.76 & 0.0 \\
\hline 24 & Oct. 27 & $21: 46$ & 1286 & 2 & 0.76 & -0.75 \\
\hline 25 & Oct. 27 & $23: 25$ & 1209 & 2 & 0.63 & -0.88 \\
\hline 26 & Oct. 28 & $7: 20$ & 1408 & 4 & 0.78 & -2.07 \\
\hline 27 & Oct. 28 & $21: 40$ & 1309 & 9 & 0.61 & -7.7 \\
\hline 28 & Oct. 28 & $23: 18$ & 1213 & 1 & 0.56 & -0.37 \\
\hline 29 & Oct. 29 & $2: 30$ & 1257 & 0 & 0.60 & 0.0 \\
\hline 30 & Oct. 29 & 4:03 & 1293 & 1 & 0.64 & -0.33 \\
\hline 31 & Oct. 29 & $5: 36$ & 1331 & 0 & 0.67 & 0.0 \\
\hline 32 & Oct. 29 & $23: 13$ & 1064 & 0 & 0.43 & 0.0 \\
\hline 33 & Oct. 30 & $0: 43$ & 1445 & 2 & 0.79 & -0.73 \\
\hline 34 & Oct. 30 & $2: 23$ & 1314 & 5 & 0.60 & -3.40 \\
\hline 35 & Oct. 30 & $18: 13$ & 1435 & 2 & 0.69 & -0.82 \\
\hline 36 & Oct. 30 & $21: 19$ & 1655 & 6 & 0.86 & -3.58 \\
\hline 37 & Oct. 30 & $22: 59$ & 1433 & 25 & 0.65 & $<-15$ \\
\hline 38 & Oct. 31 & $0: 38$ & 1357 & 12 & 0.61 & -11.47 \\
\hline 39 & Oct. 31 & $2: 01$ & 1770 & 7 & 1.11 & -3.81 \\
\hline 40 & Oct. 31 & $3: 48$ & 1428 & 5 & 0.72 & -3.06 \\
\hline 41 & Oct. 31 & $8: 40$ & 1054 & 2 & 0.44 & -1.14 \\
\hline 42 & Oct. 31 & $16: 26$ & 1210 & 3 & 0.55 & -1.73 \\
\hline 43 & Oct. 31 & 18:01 & 1569 & 1 & 0.76 & -0.27 \\
\hline 44 & Oct. 31 & $19: 39$ & 1727 & 2 & 0.87 & -0.66 \\
\hline 45 & Oct. 31 & $22: 51$ & 1568 & 2 & 0.77 & -0.75 \\
\hline 46 & Nov. 1 & $1: 55$ & 1870 & 1 & 1.19 & -0.16 \\
\hline 47 & Nov. 1 & $8: 33$ & 1093 & 0 & 0.45 & 0.0 \\
\hline 48 & Nov. 1 & $14: 41$ & 1634 & 5 & 0.74 & -3.0 \\
\hline 49 & Nov. 1 & $16: 14$ & 1341 & 3 & 0.68 & -1.49 \\
\hline 50 & Nov. 1 & $17: 53$ & 1623 & 1 & 0.81 & -0.26 \\
\hline
\end{tabular}

Oct. 30 22:59 and not outside. The count rate was halved in the data section taken during the next orbit. Thus we can essentially determine the peak count rate $A_{\text {peak }}$ and the flare decay time scale $\tau_{\text {decay }}$ (assuming an exponential flare decay) from the data; such a decay light curve with $A_{\text {peak }}=0.02 \mathrm{cts} / \mathrm{s}$ and $\tau_{\text {decay }}=0.06$ days has been overplotted in Fig. 1. Note that these number are rough estimates and we refrain from carrying out a precise error calculation.

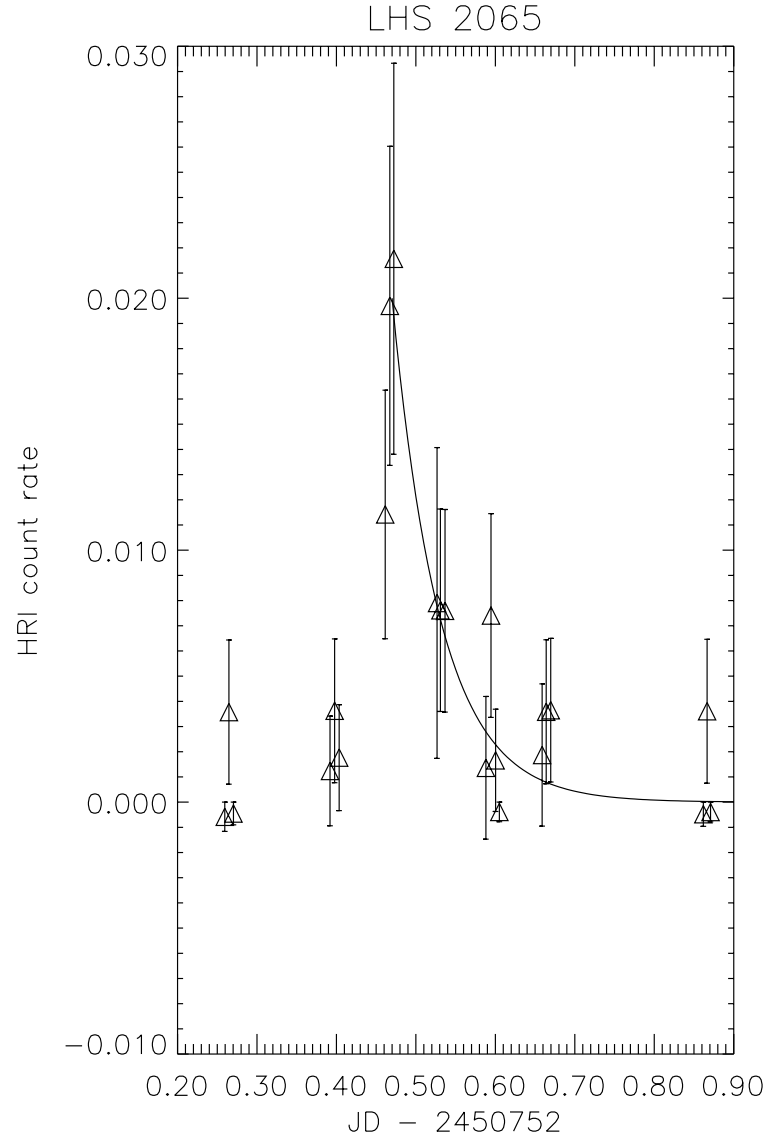

Fig. 1. Background subtracted ROSAT HRI light curve of flare on Oct. 30 1997; solid line represents an exponential decay with $A_{\text {peak }}=0.02 \mathrm{cts} / \mathrm{s}$ and $\tau_{\text {decay }}=0.06$ days.

\subsection{Quiescent $X$-ray emission}

As discussed above, the ROSAT data for LHS 2065 show clear evidence of a major flare on Oct. 30-31 1997 and a smaller flare on Oct. 28 1997. We now wish to address the question whether there is any evidence for X-ray emission outside these data intervals. As to the first part of the observation from April and May 1997, we calculate an expected total background count in the detect cell of $59.7 \mathrm{cts}$, which has to be compared with 79 observed counts. The net count of 19.3 is not significant at the " $3 \sigma$ " confidence level and we therefore consider 23.1 counts as an upper limit to any "quiescent" X-ray emission. Turning now attention to the second part of the observation taken in October and November 1997, we exclude all intervals with 6 or more detect cell counts and then find 48 counts in the detect cell compared to 17.5 expected counts. The excess of 30.5 is highly significant. We find that high significance levels apply regardless of the chosen cutoff level and conclude that during October/November observations X-ray emission was also present outside the periods with obvious flares. Whether or not this emission was "flaring" or "quiescent" is hard to tell on the basis of the existing ROSAT data. Since no clear flares are present we will call this emission "quiescent". Converting the observed count rate into apparent energy fluxes 
(using the conversion factor $2.4 \times 10^{-11} \mathrm{erg} / \mathrm{cm}^{2} /$ count) and X-ray luminosity, yields $L_{\mathrm{X} \text {,quiescent }}=2.3 \times 10^{26} \mathrm{erg} / \mathrm{s}$ for the October/November period and $L_{\mathrm{X} \text {,quiescent }}<1.8 \times$ $10^{26} \mathrm{erg} / \mathrm{s}$ for the April/May period. We therefore conclude that LHS 2065 did definitely change its X-ray properties in the two ROSAT observation intervals in spring and fall of 1997.

\section{Discussion and conclusions}

With the two measured parameters $A_{\text {peak }}$ and $\tau_{\text {decay }}$ we can address the energetics of the flare. The total number of counts - assuming an exponential decay law - expected during the decay phase is 103.7. Since we did not observe the start of the flare, we cannot make any statements about the energy release in the rise phase, but experience from many solar and stellar flares shows that the soft X-ray energy release in the rise phase is much smaller than during the decay phase. With a flux conversion factor of $2.4 \times 10^{-11} \mathrm{erg} / \mathrm{cm}^{2} /$ count we calculate an X-ray fluence of $2.5 \times 10^{-9} \mathrm{erg} / \mathrm{cm}^{2}$, which translates into a total released energy of $2 \times 10^{31} \mathrm{erg}$ with the known distance of LHS 2065. The peak X-ray luminosity was $4 \times 10^{27} \mathrm{erg} / \mathrm{s}$, which corresponds to an emission measure of $2 \times 10^{50} \mathrm{~cm}^{-3}$ assuming a cooling function value of $2 \times 10^{-23} \mathrm{erg} \mathrm{cm}^{3} / \mathrm{s}$. We mention in passing that the HRI data do not allow us to determine the X-ray temperature of the flare plasma; we assume the plasma to have the "usual" temperature of $10^{7} \mathrm{~K}$. Note that at flare peak the X-ray luminosity of LHS 2065 exceeds that of the quiescent Sun at solar maximum, and the total soft X-ray energy release is at least within an order of magnitude of the largest observed solar flares. During the flare the $L_{\mathrm{X}} / L_{\mathrm{bol}}$ ratio exceeds the saturation level of $10^{-3}$, but note that this refers to the quiescent $L_{\text {bol }}$ value.

The decay time of the flare is related to the characteristic plasma density $N_{\mathrm{e}}$ through the formula (cf. Schmitt et al. 1987)

$\tau_{\text {decay }}=\frac{3 k T}{N_{\mathrm{e}} P(T)}$,

where $P(T)$ denotes the radiative cooling function and $k$ Boltzmann's constant. This formula assumes pure radiative cooling; of course, the flare may also cool conductively, in which case even larger densities apply. Hence Eq. (1) provides a lower limit for the density. Evaluating (1) with the measured value of $\tau_{\text {decay }}$ and assuming $T=10^{7} \mathrm{~K}$ results in $N_{\mathrm{e}} \sim 4 \times 10^{10} \mathrm{~cm}^{-3}$, i.e., a plasma density quite typical for solar flares. Assuming then a "canonical" loop with an aspect ratio 0.1 we find a loop length of $1.6 \times 10^{10} \mathrm{~cm}$. This length is small compared to a solar radius, but is on a scale comparable to the radius of LHS 2065. We may speculate that the flaring loop connects the two poles of the star with opposite polarity, however, one must keep in mind that the density values are inferred from rather uncertain assumptions and the true length scales may be smaller.
The X-ray flare observed on LHS 2065 provides evidence further that very late-type stars can produce rather vigorous coronal energy releases. Presumably these events are linked to the strong $\mathrm{H}_{\alpha}$-emission events discussed by Martín \& Ardila (2001); the observed one strong X-ray flare is certainly consistent with their estimated rate of $<0.03 \mathrm{hr}^{-1}$ for strong $\mathrm{H}_{\alpha}$-flares. We are not aware of white light flare observations of LHS 2065. Assuming - arbitrarily - a $B$-band flux equal to the observed soft X-ray flux, LHS 2065 should attain a magnitude of at least 18 in the $U, B$ and $V$ bands during the flare, a magnitude detectable even with smaller telescopes.

The ROSAT observations of LHS 2065 add to the more and more increasing evidence that possibly all ultracool stars may experience at least transient X-ray emission. In the ROSAT data there is in addition a case for X-ray emission outside obvious flare events, but the question of whether or not quiescent X-ray emission really exists in ultracool stars and brown dwarfs is a pressing issue that needs to be addressed with the currently available generation of more sensitive new X-ray telescopes.

Acknowledgements. Use of the SIMBAD database operated by the CDS, Strasbourg, and the ROSAT data archive run by MPE is acknowledged.

\section{References}

Bailer-Jones, C. A. L., \& Mundt, R. 1999, A\&A, 348, 800

Basri, G., \& Marcy, G. W. 1995, AJ, 109, 762

Basri, G. 2000, ARA\&A, 38, 485

Berger, E., Ball, S., Becker, K. M., et al. 2001, Nature, 410, 338

Fleming, T. A., Giampapa, M. S., Schmitt, J. H. M. M., \& Bookbinder, J. A. 1993, ApJ, 410, 387

Fleming, T. A., Giampapa, M. S., \& Schmitt, J. H. M. M. 2000, ApJ, 533, 372

Fleming, T. A., et al., Proceedings of Stellar Coronae 2001, ed. F. Favata, \& J. Drake (Noordwij)

Giampapa, M. S., \& Liebert, J. 1986, ApJ, 305, 784

Gizis, J. E., Monet, D. G., Reid, I. N., et al. 2000, AJ, 120, 1085

Hawley, S. L., Gizis, J. E., \& Reid, I. N. 1996, AJ, 112, 2799

Liebert, J., Kirkpatrick, J. D., Reid, I. N., \& Fisher, M. D. 1999, ApJ, 519, 345

Martín, E. L., \& Ardila, D. R. 2001, AJ, 121, 2758

Monet, D. G, Dahn, C. C., Vrba, F. J., et al. 1992, AJ, 103, 638

Neuhäuser, R., \& Comerón, F. 1999, Science, 282, 83

Neuhäuser, R., Briceño, C., Comerón, F., et al. 1999, A\&A, 343,883

Preibisch, T., \& Zinnecker, H. 2001, AJ, in press

Reid, I. N., Kirkpatrick, J. D., Gizis, J. E., \& Liebert, J. 1999, ApJ, 527, L105

Rutledge, R. E., Basri, G., Martín, E. L., \& Bildsten, L. 2000, ApJ, 538, L141

Schmitt, J. H. M. M., Fink, H., \& Harnden, F. R. 1987, ApJ, 322,1023

Schmitt, J. H. M. M., Fleming, T. A., \& Giampapa, M. S. 1995, ApJ, 450, 392

Schneider, D. P., Greenstein, J. L., Schmidt, M., \& Gunn, J. E., 1991, AJ, 102, 1180 